timepoint analysis was more affected. With only 2 readings, sensitivity was reduced for all scores. Specificity was unaffected by data reduction (Table 1).

\begin{tabular}{|c|c|c|c|}
\hline & Oasys score & $A B C$ score & Timepoint analysis \\
\hline Pre-Randomisation & $75(84)$ & 69 (93) & $64(84)$ \\
\hline Randomisation 1 & $67(73)$ & $61(89)$ & $58(84)$ \\
\hline Randomisation 2 & $72(68)$ & $55(86)$ & $44(86)$ \\
\hline Randomisation 3 & $86(86)$ & $69(93)$ & $53(86)$ \\
\hline$\geq 7$ readings per day & $73(86)$ & $65(95)$ & $62(86)$ \\
\hline 6 readings per day & $73(80)$ & $65(100)$ & $58(81)$ \\
\hline 5 readings per day & $69(84)$ & $65(97)$ & $62(84)$ \\
\hline 4 readings per day & $73(84)$ & $69(97)$ & $54(84)$ \\
\hline 3 readings per day & $69(81)$ & $65(97)$ & $50(81)$ \\
\hline 2 readings per day & $50(86)$ & $34(97)$ & 27 (91) \\
\hline
\end{tabular}

Conclusion Specificity was not reduced by adding random errors to the peak flow measurements nor through data reduction. Sensitivity was reduced, relatively more for the timepoint analysis, but in $2 / 3$ randomisations it was preserved for the Oasys and $\mathrm{ABC}$ systems. Oasys analysis is robust despite decreasing data quality and quantity.

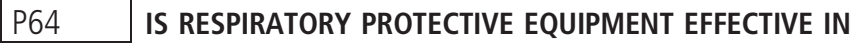 ABOLISHING THE WORK EFFECT IN PATIENTS SENSITISED TO METAL WORKING FLUID?}

${ }^{1}$ A llgaz, ${ }^{1}$ VC Moore, ${ }^{2}$ W Robertson, ${ }^{3}$ AS Robertson, ${ }^{1} P S$ Burge. ${ }^{1}$ Birmingham Heartlands Hospital, Birmingham, UK; ${ }^{2}$ University of Warwick, Coventry, UK; ${ }^{3}$ University Hospitals Bimringham, Birmingham, UK

\subsection{6/thoraxjn-2015-207770.201}

Background Removing exposure to the causative agent in workers with occupational asthma or hypersensitivity pneumonitis is the main goal. The effectiveness of Respiratory Protective Equipment (RPE) in sensitised workers with usual exposures in their workplace is not known. We aimed to assess changes in serial peak expiratory flow (PEF) in workers with sensitisation to metal working fluid before and after the introduction of RPE.

Methods Workers who had been requested to keep 2-hourly PEF measurements before and after the introduction of RPE at a particular engine manufacturing plant were searched for on the Oasys PEF database. Those who had completed a minimum of 1 week before and after the introduction of RPE and had a positive area between curves (ABC) score for occupational effect from Oasys analysis $(\geq 15 \mathrm{~L} / \mathrm{min} / \mathrm{hr})$ on either record were included. All PEF measurements were made outside the working area where the RPE could be removed. Workers who remained symptomatic after cleaning and replacement of the metal-working fluid were only allowed to continue work if they complied with the strict use of respiratory protective equipment (RPE) using powered filtration and external changing facilities.

Results 19 workers fulfilled the inclusion criteria. The mean ABC score before RPE use was $27.85 \mathrm{~L} / \mathrm{min} / \mathrm{hr}$ (SD 17.69) and after RPE use was $19.65 \mathrm{~L} / \mathrm{min} / \mathrm{hr}$ (SD 22.77) showing a mean overall decrease of $8.20 \mathrm{~L} / \mathrm{min} / \mathrm{hr}$ (SD 28.60). 9 workers (47\%) no longer showed an occupational effect on their PEF record once RPE was instated, 7 workers continued to show an occupational effect and 3 workers showed a new occupational effect which was not present before RPE use.

Conclusion Serial PEF measurements assessed with the ABC score from the Oasys system allowed quantification of the effect of RPE in sensitised workers. The RPE abolished falls in PEF associated with work exposure in $47 \%$ of workers, the remaining 10 workers had significant falls in PEF despite rigorously applied RPE. RPE should still remain a last resort in the hierarchy of control for occupational health.

\section{P65 RESPIRATORY ILL HEALTH IN THE SILICA EXPOSED STONE WORKING SECTOR}

D Fishwick, J Sumner, CM Barber, E Robinson, A Codling, L Lewis, C Young, N Warren. Centre for Workplace Health, HSL, Buxton, UK

\subsection{6/thoraxjnl-2015-207770.202}

Introduction Exposure at work to inhaled respirable crystalline silica (RCS) has previously been linked with silicosis, tuberculosis, lung cancer and COPD. Whilst the risk of developing silicosis is largely a function of cumulative lifetime RCS exposures, current workplace exposures contribute to this risk. New cases of silicosis continue to be reported in the UK. The stone working sector is one where exposures to RCS continue to place workers at risk.

Methods A cross sectional GB based workplace study of stone workers was carried out, in order to identify a subsequent longitudinal cohort. Consenting workers were asked to complete an interviewer led questionnaire, undergo lung function testing and complete a full occupational history including details of lifetime exposure to RCS. Consenting workers had a PA Chest Radiograph using a mobile facility, and levels of RCS exposure in the personal breathing zone were measured.

Results 128 workers took part; with a mean age of 40.1 years and 11 years median (range 0.5-44) years worked overall in industry. One had radiological evidence of silicosis. Respiratory symptoms were common; for example $22.7 \%$ reported cough, $33.6 \%$ wheeze in the last 12 months, $16.4 \%$ reported ever having asthma. $14.1 \%$ reported at least one work related respiratory symptom. Mean lung function values $(\mathrm{n}=127)$ were as follows; mean (SD) percentage predicted $\mathrm{FEV}_{1} 97.5$ (14.5) and FVC 103.6 (12.2).

Twenty four workers had measured airways obstruction (as defined by an $\left.\mathrm{FEV}_{1} / \mathrm{FVC}<0.7\right)$; in this cross sectional analysis its presence did not significantly relate to current smoking status or duration of RCS exposure, although was associated with the reporting of a diagnosis of (ever having) asthma or chest tightness and difficulty in breathing.

Conclusions This cross sectional study of stone workers has identified a cohort for longer term follow up. Future work will allow the development dose response relationships (using measured current, and historic, RCS levels) corrected for other relevant factors, between cumulative RCS exposure and $\mathrm{FEV}_{1}$ decline. These approaches will assist in the development of future workplace interventions to reduce the health risks associated with RCS exposure in stone workers. 